\title{
Design of Slotted Single-Mode Lasers Suitable for Photonic Integration
}

\author{
Qiaoyin Lu, Wei-Hua Guo, Diarmuid Byrne, and John F. Donegan, Senior Member, IEEE
}

\begin{abstract}
A single-mode laser structure suitable for photonic integration is presented. The laser can be fabricated by standard photolithography without any regrowth steps. Through optimization, a threshold current as low as $13 \mathrm{~mA}$ and a sidemode suppression ratio (SMSR) up to $47 \mathrm{~dB}$ have been predicted for a $400-\mu \mathrm{m}$ -long laser. A high yield up to $90 \%$ is also predicted with the requirement that the SMSR is higher than $40 \mathrm{~dB}$ at an injection current of $100 \mathrm{~mA}$.
\end{abstract}

Index Terms-Sidemode suppression ratio (SMSR), single-mode laser, surface grating.

\section{INTRODUCTION}

W AVELENGTH-STABILIZED single-mode semiconductor lasers have found wide application in optical communications. Distributed-feedback (DFB) lasers as typical single-mode lasers have achieved great success. However, fabricating these lasers requires both high-resolution processing and complex regrowth steps. Laterally coupled DFB lasers [1], [2] and surface-grating distributed Bragg reflector (DBR) lasers [3] which use just a single growth step have thus attracted great interest. However, these devices generally need electron-beam lithography for patterning which is quite expensive compared with the standard photolithography. On the other hand, single-mode semiconductor lasers can also be realized by introducing reflective defects (slots) into conventional Fabry-Pérot (FP) laser cavities [4]-[8]. By carefully optimizing the slot positions and slot number, lasing with a sidemode suppression ratio (SMSR) of more than $40 \mathrm{~dB}$ has been achieved [6], [7]. However, these lasers need both cleaved facets to form the fundamental FP cavity so they cannot be employed to integrate monolithically with other optical devices such as electroabsorption (EA) modulators. By increasing the slot depth, we have recently demonstrated a discretely tunable laser which completely relies on these slots to provide necessary reflectivity for the laser operation independent of any cleaved facets [9]. However, they are not suitable as single-mode lasers because the very long laser cavity will result in a very high laser threshold. A laser based on such deep slots integrated with a photodetector has also been presented [10]; however, the laser suffered from a low SMSR due to the broad reflection

Manuscript received December 18, 2009; revised February 17, 2010; accepted March 08, 2010. Date of publication March 22, 2010; date of current version May 07, 2010. This work was supported by SFI PIFAS under Grant 07/SRC/I1173.

The authors are with the Semiconductor Photonics Group, School of Physics, Trinity College, Dublin 2, Ireland (e-mail: luqi@tcd.ie; guow@tcd.ie; byrnedc@tcd.ie; jdonegan@tcd.ie).

Color versions of one or more of the figures in this letter are available online at http://ieeexplore.ieee.org.

Digital Object Identifier 10.1109/LPT.2010.2045888

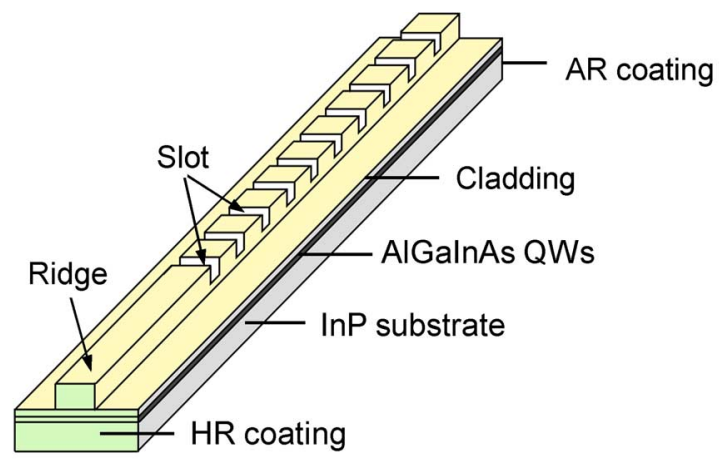

(a)

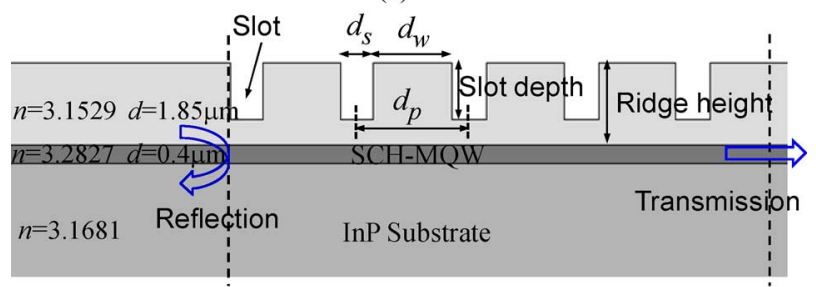

(b)

Fig. 1. (a) Three-dimensional schematic structure of the slotted single-mode laser. (b) Simplified 2-D waveguide structure with the slot.

bandwidth caused by the small number of deep slots. In this letter, we present the design of a single-mode laser which relies on slots on one end of the laser to provide the reflection so it is suitable for monolithic integration with other optical devices such as the EA modulators. Through optimization, we demonstrate that the single-mode laser can provide a threshold current of $13 \mathrm{~mA}$ and an SMSR up to $47 \mathrm{~dB}$ and output slope efficiency of $0.21 \mathrm{~mW} / \mathrm{mA}$ from the slot section side.

\section{NUMERICAL SimULATION}

The schematic structure of the slotted laser is shown in Fig. 1(a), which is a typical $2.0-\mu \mathrm{m}$-wide ridge waveguide laser. The laser has the same epitaxial structure as in [10]. The active layer consists of five AlGaInAs quantum wells which have an emission peak around $1545 \mathrm{~nm}$. One side of the laser has multiple uniformly distributed slots which act as an active DBR of the laser. The facet at the end of the slot region is antireflection (AR)-coated (no reflection is assumed in the following simulation). The other end of the laser has a high-reflection (HR)-coated facet $(90 \%$ power reflection assumed). The laser has a single contact which covers the whole laser cavity including the group of slots, so the regions with and without slots will have the same current injection. The shift of the reflection peak with current injection will not influence the SMSR because the longitudinal mode position will shift simultaneously. The influence of injected current on 


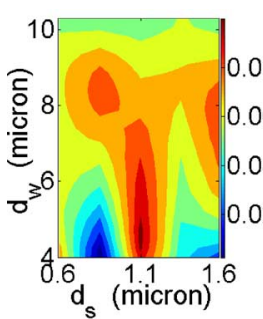

(a)

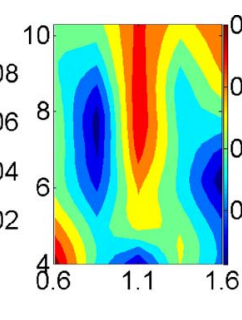

(b)

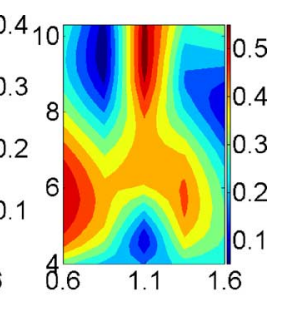

(c)
Fig. 2. Contour plot of simulated amplitude reflection versus slot width and spacing for 20 slots with the slot depth of (a) 1.0 , (b) 1.35 , and (c) $1.6 \mu \mathrm{m}$.

the emitting wavelength of this laser will be the same as the influence on a DFB laser.

Under the effective index approximation, the three-dimensional (3-D) ridge waveguide is simplified into a two-dimensional (2-D) structure as shown in Fig. 1(b), where the waveguide core layer has an average refractive index of 3.2827 with a thickness of $0.4 \mu \mathrm{m}$. The top InP layer of $1.85 \mu \mathrm{m}$ has an effective index of 3.1529 in the 2-D situation. The slot parameters such as the slot width $d_{s}$, spacing $d_{w}$, depth, and slot number are key parameters which influence the laser performance and have to be optimized in the design.

In order to determine the optimum geometrical parameters of the slots, the scattering matrix method (SMM) [11] is used for the simulation, and the interactions between these slots are accounted properly by applying the SMM method directly in 2-D. To investigate the effects of the slot width and spacing on the reflection and transmission of a group of slots, we initially take 20 slots with uniform period to analyze using the SMM method. Three slot depths of 1.0, 1.35, and $1.6 \mu \mathrm{m}$ from shallow to deep are considered. In the simulation, the slot width and slot spacing are defined as $d_{s}=(2 p+1) \lambda_{B} / 4 n_{s}$ and $d_{w}=(2 q+1) \lambda_{B} / 4 n_{w}$, respectively, where $p$ and $q$ are integers, $n_{s}$ and $n_{w}$ are the effective index of the slot region and the waveguide region, $\lambda_{B}$ is the Bragg wavelength. Because of this definition, even when the slot width and spacing changes, the Bragg wavelength does not change. In the simulation, the Bragg wavelength is always set to be $1550 \mathrm{~nm}$. We are interested in the slot width around $1 \mu \mathrm{m}$ that can be easily fabricated by standard photolithography. The contour plot of the calculated amplitude reflection is given in Fig. 2, which shows that the slot width and spacing influence the reflection strongly. It is also observed that when the slot width is around $1.1 \mu \mathrm{m}$ (in this case, the slot can be fabricated by standard photolithography), there exists high reflection peaks for these three slot depths, and slots with deeper depth need larger spacing for maximizing the reflection. For slots with a depth of $1.35 \mu \mathrm{m}$, the optimized slot width and slot depth are around 1.1 and $7.87 \mu \mathrm{m}$, respectively, which makes the slot section act as a 37th-order surface grating.

By increasing the slot number a sufficiently high reflection can be obtained but with a sacrifice of the transmission due to the loss caused by each slot, as shown in Fig. 3(a) and (b). For deep slots due to the relatively large scattering loss, the reflection will saturate quickly with the slot number increase. For a slot depth of $1.6 \mu \mathrm{m}$, the reflection starts to saturate at a slot number of 10 , while the transmission continues to drop with the slot number increase. For slots with a shallow depth of $1.0 \mu \mathrm{m}$, due to the reflection from a single slot being relatively weak, a group of
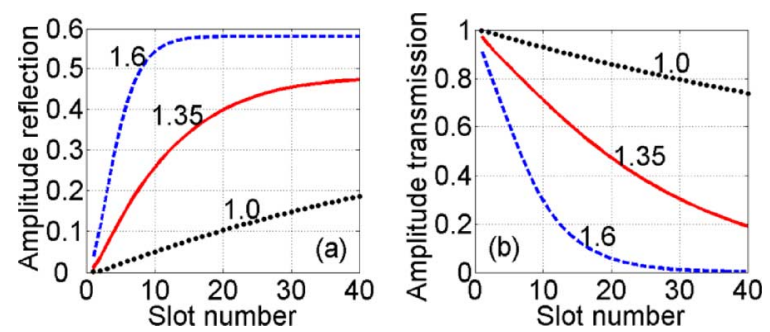

Fig. 3. Calculated amplitude reflection and transmission from a group of slots with the slot depth of $1.0,1.35$, and $1.6 \mu \mathrm{m}$, respectively, versus the slot number for (a) reflection and (b) transmission.
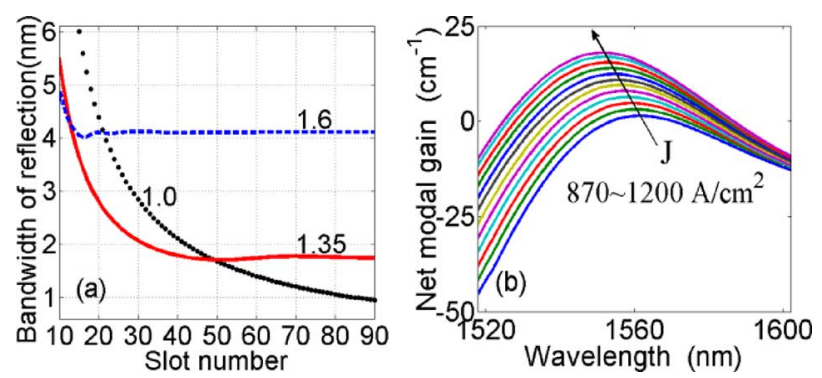

Fig. 4. (a) Calculated bandwidth of reflection peaks from a group of slots with the slot depth of 1.0,1.35, and $1.6 \mu \mathrm{m}$ versus the slot number. (b) Measured net modal gain spectrum for the laser wafer structure.

40 slots just provides an amplitude reflection of about 0.2 , which could necessitate a long laser cavity resulting in a high laser threshold. For a slot depth of around $1.35 \mu \mathrm{m}$, the reflection of 24 slots is around 0.43 with a transmission around 0.4.

A relatively narrow reflection spectrum is generally required to ensure a good single-mode operation. Fig. 4(a) shows the $3-\mathrm{dB}$ bandwidth of the reflection spectrum versus the slot number for different slot depths. It can be seen that the 1.6- $\mu \mathrm{m}$ slots will have a relatively broad reflection spectrum with the $3-\mathrm{dB}$ bandwidth around $5 \mathrm{~nm}$ for 10 slots, which will result in a poor SMSR. For the slot depth around $1.35 \mu \mathrm{m}$, when the slot number is between 20 and 30, a reflection bandwidth narrower than $3 \mathrm{~nm}$ can be obtained, which would cover two to three longitudinal modes of a conventional FP laser with a $300-\mu \mathrm{m}$ cavity length. The bandwidth for the $1.0-\mu \mathrm{m}$ depth case is continuously dropping with slot number increase, and will be narrower than $1 \mathrm{~nm}$ when the slot number reaches 90 .

From the above analysis, for the trade-off between maximizing the reflectivity and minimizing the bandwidth of the reflection peaks while also ensuring that the laser cavity length is kept to a minimum, the slots are optimized with a slot number of 24 and slot depth of $1.35 \mu \mathrm{m}$ which is much smaller than the $1.85-\mu \mathrm{m}$ ridge height. For such a group of slots, an amplitude reflection and transmission around 0.43 and 0.4 , respectively, can be generated, and the bandwidth is around $2.5 \mathrm{~nm}$ just covering three modes of the laser with an effective cavity length of around $300 \mu \mathrm{m}$. This helps to achieve good single-mode operation with a minimum laser threshold. By measuring the FP laser based on the same wafer structure, we obtain the net modal gain spectrum as shown in Fig. 4(b), and the internal loss and the injection efficiency are measured to be around $22 \mathrm{~cm}^{-1}$ and 0.6 , respectively. These parameters are used to analyze the optimized slotted laser. The slot region of the laser will have current injected, i.e., gain will be induced in the slot 

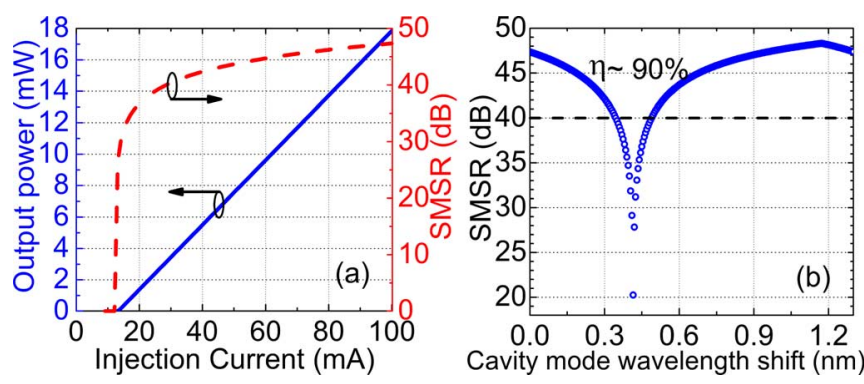

Fig. 5. (a) Simulated output power and SMSR versus injected current for the optimized slotted laser structure. (b) Simulated SMSR versus the cavity mode wavelength shift.
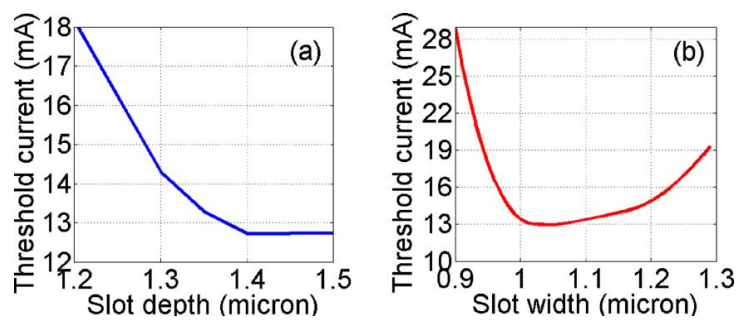

Fig. 6. Simulated threshold current versus (a) slot depth and (b) slot width variations caused by practical fabrication imperfections.

region, which will increase the reflection and transmission from the group of slots. This has been taken into account in the following simulation for the laser performances (threshold current, output power, and SMSR). By scanning the dependence of the laser threshold on the total cavity length, we optimize the total cavity length to be around $400 \mu \mathrm{m}$ to minimize the threshold. By solving the multimode rate equations the output laser characteristics can be analyzed.

Fig. 5(a) shows the simulated light output-current $(L-I)$ curve as well as the SMSR versus current for the optimized laser structure. It can be seen that a low threshold current of about $13 \mathrm{~mA}$ and a slope efficiency of about $0.21 \mathrm{~mW} / \mathrm{mA}$ from the slot side can be obtained. A stable single-mode operation with SMSR up to $47 \mathrm{~dB}$ at an injection level of $100 \mathrm{~mA}$ is also predicted. Since the laser still needs one cleaved facet to work, there is a yield issue caused by the uncertainty of the cleaved facet position assuming the laser is perfectly fabricated. For the laser with the total cavity length of $400 \mu \mathrm{m}$, the penetration of these 24 slots (period $8.97 \mu \mathrm{m}$ ) is around $103 \mu \mathrm{m}$ from the equation of $(N-1) d_{\mathrm{p}} / 2$ [9] [where $d_{p}$ is the period as shown in Fig. 1(b)]. Thus the effective cavity length is around $288 \mu \mathrm{m}$ which results in a longitudinal mode spacing about $1.3 \mathrm{~nm}$. By simulating the cavity mode wavelength shifting in the range of one mode spacing, we can calculate this yield of the designed laser. The calculated SMSR versus the cavity mode wavelength shift is shown in Fig. 5(b). It is seen that a high yield of around $90 \%$ can be obtained with the requirement that the SMSR is higher than $40 \mathrm{~dB}$ at a $100-\mathrm{mA}$ injected current.

The fabrication tolerances of the slot parameters are also simulated. Fig. 6(a) shows the calculated threshold current versus the slot depth varying from 1.2 to $1.5 \mu \mathrm{m}$, which shows that a proper etching stop layer should be included within the wafer structure to properly control the slot etching depth and a relatively deep etching depth is preferred to ensure a low threshold.
Fig. 6(b) shows the simulated laser threshold versus the varied slot width assumed to be caused by the fabrication inaccuracy. It is assumed that the center-to-center slot period is unchanged during fabrication. It can be seen that a variation of $\pm 0.1 \mu \mathrm{m}$ will not cause any big increase on the laser threshold. Since one feedback of the laser is completely dependent on the group of slots, it is suitable to monolithically integrate this laser with other optical devices such as semiconductor optical amplifiers (SOAs), EA modulators, etc.

\section{CONCLUSION}

In summary, we have designed a slotted single-mode laser structure suitable for photonic integration. Through optimization a laser threshold as low as $13 \mathrm{~mA}$ and an SMSR up to $47 \mathrm{~dB}$ has been predicted. A high yield up to $90 \%$ is also predicted with the requirement that the SMSR is higher than $40 \mathrm{~dB}$ at an injection current of $100 \mathrm{~mA}$. The fabrication tolerances of the slot depth and slot width are also analyzed. The presented laser structure which just needs a single wafer growth, can be easily fabricated by standard photolithography and should provide a significant cost advantage.

\section{REFERENCES}

[1] L. M. Miller, J. T. Verdeyen, J. J. Coleman, R. P. Bryan, J. J. Alwan, K. J. Beernink, J. S. Hughes, and T. M. Cockerill, "A distributed feedback ridge waveguide quantum well heterostructure laser," IEEE Photon. Technol. Lett., vol. 3, no. 1, pp. 6-8, Jan. 1991.

[2] R. D. Martin, S. Forouhar, S. Keo, R. J. Lang, R. G. Hunsperger, R. C. Tiberio, and P. F. Chapman, "CW performance of an InGaAs-GaAs-AlGaAs laterally coupled distributed feedback (LC-DFB) ridge laser diode," IEEE Photon. Technol. Lett., vol. 7, no. 3, pp. 244-246, Mar. 1995

[3] R. M. Lammert, J. S. Hughes, S. D. Roh, M. L. Osowski, A. M. Jones, and J. J. Coleman, "Low-threshold narrow-linewidth InGaAs-GaAs ridge-waveguide DBR lasers with first-order surface gratings," IEEE Photon. Technol. Lett., vol. 9, no. 2, pp. 149-151, Feb. 1997.

[4] B. Corbett and D. McDonald, "Single longitudinal mode ridge waveguides $1.3 \mu \mathrm{m}$ Fabry-Perot laser by modal perturbation," Electron. Lett., vol. 31, pp. 2181-2182, 1995.

[5] J. Patchell, D. Jones, B. Kelly, and J. O'Gorman, "Specifying the wavelength and temperature tuning range of a Fabry-Perot laser containing refractive index perturbations," Proc. SPIE., vol. 5825, pp. 1-13, 2005.

[6] S. O'Brien and E. P. O'Reilly, "Theory of improved spectral purity in index patterned Fabry-Perot lasers," Appl. Phys. Lett., vol. 86, pp. 201101-1-201101-3, 2005.

[7] B. Kelly, R. Phelan, D. Jones, C. Herbert, J. O'Carroll, M. Rensing, J. Wendelboe, C. B. Watts, A. Kaszubowska-Anandarajah, P. Perry, C. Guignard, L. P. Barry, and J. O'Gorman, "Discrete mode laser diodes with very narrow linewidth emission," Electron. Lett., vol. 43, pp. 1282-1284, 2007.

[8] R. Phelan, W. -H. Guo, Q. Y. Lu, D. Byrne, B. Roycroft, P. Lambkin, B. Corbett, F. Smyth, L. P. Barry, J. Patchell, B. Kelly, J. O'Gorman, and J. F. Donegan, "A novel two-section tunable discrete mode Fabry-Pérot laser exhibiting nanosecond wavelength switching," IEEE J. Quantum Electron., vol. 44, no. 4, pp. 331-337, Apr. 2008.

[9] D. C. Byrne, J. P. Engelstaedter, W. H. Guo, Q. Y. Lu, B. Corbett, B. Roycroft, J. O'Callaghn, F. H. Peters, and J. F. Donegan, "Discretely tunable semiconductor lasers suitable for photonic integration," IEEE J. Sel. Topics Quantum Electron., vol. 15, no. 3, pp. 482-487, May/Jun. 2009.

[10] J. P. Engelstaedter, B. Roycroft, and B. Corbett, "Laser and detector using integrated reflector for photonic integration," Electron. Lett., vol. 44, pp. 1017-1019, 2008.

[11] Q. Lu, W. Guo, R. Phelan, D. Byrne, J. F. Donegan, P. Lambkin, and B. Corbett, "Analysis of slot characteristics in slotted single-mode semiconductor lasers using the 2-D scattering matrix method," IEEE Photon. Technol. Lett., vol. 18, no. 24, pp. 2605-2607, Dec. 15, 2006. 